THE SECOND CAPE PHOTOGRAPHIC CATALOGUE: CPC2

\author{
C.A. MurRay, M.J. Penston \\ Royal Greenwich Observatory \\ Herstmonceux Castle \\ Hailsham, E. Sussex BN27 1RP, U.K. \\ and \\ N. Zacharias, Chr. DE VegT \\ Hamburger Sternwarte \\ D-2050 Hamburg \\ Germany, Fed.Rep.
}

ABSTRACT. Between 1962 and 1972 the southern hemisphere has been covered by a 4-fold overlap pattern on 5820 plates, taken with a scale of $100 \mathrm{arcsec} / \mathrm{mm}$ and using a visual bandpass. More than 2 million pairs of $x, y$ coordinates of the 2 exposures from 276259 stars have been measured at RGO with the GALAXY machine. The conventional plate adjustment, carried out at Hamburg Observatory, is now complete. About 150000 primary stars will achieve a catalogue accuracy of about 0.06 arcsec at epoch of observation.

The rigorous FK4 and FK5 versions, using the SRS catalogue (C. Smith 1988), will be supplemented by preliminary new proper motions for those stars common with the SAOC. Finally, a rigorous block adjustment will be performed within the next year.

\title{
AN ASTROMETRIC CATALOG OF FOUR MILLION STARS
}

\author{
V.V. Nesterov, V.S. KisLiuK, H.I. PotTeR \\ Central Astronomical Observatory \\ Pulkovo \\ 196140 Leningrad, USSR
}

ABSTRACT. The goal of the present study is

(1) Completion of a general reduction of the Carte $d u$ Ciel astrophotographic catalogs, measurement of the positions in them on modern astronegatives, derivation of proper motions and compilation of a catalog of stars to magnitude 12.5 .

(2) Copying the data of all 272 volumes of astrophotographic catalogues onto magnetic tapes (all together 8.5 million measurements).

(3) Measurement of modern astronegatives on FON-OBZOR-S and FOKAT-Y programs.

(4) Studies of the magnitude equation of the AGK3 catalog and other catalogs which will be presumably used as reference catalogs.

(5) Development and comparative studies of several methods of determination of equatorial coordinates on the basis of full information on astronegative measurements. Application of these methods to compilation of the catalog of 4 million stars. 